\title{
¿Cómo desarrollar la autoeficacia del estudiantado? Presentación y evaluación de una experiencia formativa en el aula de traducción
}

How to develop students' self-efficacy? Description and evaluation of a learning experience in the translation classroom

Como desenvolver a autoeficacia do alunado? Apresentação e avaliação de uma experiência formativa na aula de tradução

María del Mar Haro Soler* (https://orcid.org/0000-0001-8467-8932)

Departamento de Traducción e Interpretación, Universidad de Granada, Granada, España
Recibido: 29-09-17

Revisado: $31-10-17$

Aceptado: $23-11-17$

Publicado: 18-12-17

RESUMEN. Ante el vacío existente en la formación de traductores en torno al desarrollo estructurado de la autoeficacia del estudiantado (Atkinson \& Crezee, 2014), se presenta en este artículo una experiencia formativa implementada en el aula universitaria de traducción con el objetivo de facilitar la confianza que el estudiantado posee en sus capacidades para traducir, o autoeficacia para traducir (Bandura, 1987, 1997; Haro-Soler, 2017a, 2017b). Dicha experiencia incluyó prácticas tan variadas como el pensamiento autorreferente, la persuasión verbal, el aprendizaje vicario o el establecimiento de objetivos que funcionan

Palabras clave: enseñanza y formación, traducción, competencias, autoeficacia,

Educación Superior como incentivos motivacionales. Se presentarán, además, los resultados del estudio empírico-descriptivo desarrollado con el objetivo general de evaluar esta experiencia formativa desde la perspectiva de los 15 estudiantes que participaron en ella. Para ello, se recurrió a la técnica de la encuesta, que se materializó en un cuestionario en línea que arrojó datos tanto de corte cuantitativo como cualitativo. De acuerdo con los resultados obtenidos, los participantes consideran que la experiencia implementada resulta de utilidad para su formación como traductores y sugieren que este tipo de experiencias formativas, destinadas a favorecer su autoeficacia, deberían incorporarse a la formación de traductores en particular y a la Educación Superior en general.

Citar como: Haro-Soler, M. M. (2017). ¿Cómo desarrollar la autoeficacia del estudiantado? Presentación y evaluación de una experiencia formativa en el aula de traducción. Revista Digital de Investigación en Docencia Universitaria, 11(2), 50-74. doi: http://dx.doi.org/10.19083/ridu.11.567

*E-mail: mmarharosolerQugr.es 
ABSTRACT. This paper aims at filling the gap regarding the need to incorporate selfefficacy in translator training programmes in a structured way (Atkinson \& Crezee, 2014). It describes a learning experience implemented in the translation classroom aimed at developing translation students' self-efficacy, i.e., their confidence in their ability to translate (Bandura 1987, 1997; Haro-Soler, 2017a, 2017b). This experience included practices such as goal-setting as a motivational incentive, verbal persuasion, vicarious learning, and activities to promote students' self-awareness. The results of an empirical-descriptive study performed to qualitatively and quantitatively assess this learning experience from the participants' perspective will also be presented. The results were obtained from an online questionnaire that all 15 participants were asked to complete and showed that, in their opinion, the learning experience implemented was useful for their training as translators. Participants also suggested that initiatives intended to help students trust their abilities should be incorporated not only into translator training but in higher education programs in general.

RESUMO. Diante da brecha existente na formação de tradutores no que diz respeito ao desenvolvimento da autoeficácia do alunado (Atkinson y Crezee, 2014), este artigo relata a experiência formativa implementada na aula universitária de tradução com o objetivo de aumentar a confiança que os estudantes têm nas suas capacidades de traduzir, a autoeficácia para traduzir (Bandura, 1987, 1997; Haro-Soler, 2017a, 2017b). Tal experiência incluiu variadas práticas como o pensamento autorreferente, a persuasão verbal, a aprendizagem por imitação ou o estabelecimento de objetivos que funcionam como incentivos motivacionais. Apresentam-se, além dos resultados do estudo empíricodescritivo desenvolvido com o objetivo geral de avaliar esta experiência formativa dos 15 estudantes que participaram dela. Para isso se realizou uma pesquisa que resultou em um questionário on-line fornecendo dados tanto para o corte quantitativo como qualitativo. De acordo com os resultados obtidos, os participantes consideram que a experiência implementada é útil para a sua formação como tradutores sugerindo que este tipo de experiências formativas, destinadas a aumentar a autoeficácia, deveriam incorporar-se à formação de tradutores em particular e a Educação Superior em geral.

\author{
Keywords: \\ teaching \\ and training, \\ translation, \\ competence, \\ self-efficacy, \\ higher \\ education
}

Palavraschave:

ensino e

formação,

tradução,

competências, auto eficácia,

Educação Superior

Si bien es fácil pensar que el término autoeficacia se refiere a la capacidad de un individuo para lograr el rendimiento deseado, Bandura (1987), quien hizo mención por primera vez a la autoeficacia en 1977 con su publicación Self-Efficacy: Toward a Unifiying Theory of Behavioural Change, especifica que dicho término no hace referencia a las capacidades que un individuo posee, sino a lo que se considera capaz de lograr gracias a ellas. Más concretamente, define este término como “los juicios de cada individuo sobre sus capacidades, en base a los cuales organizará y ejecutará sus actos de modo que le 
permitan alcanzar el rendimiento deseado" (Bandura, 1987, p. 416). Subraya, además, que dicho juicio se refiere a la tarea o actividad específica que el individuo desea desarrollar (Bandura, 2006, p. 307). Nos encontramos, por tanto, ante una autopercepción de las propias capacidades para llevar a cabo una tarea particular o, dicho de otro modo, a "la confianza que tiene una persona de que posee la capacidad para hacer las actividades que trata de hacer" (Blanco, Martínez, Zueck \& Gastélum, 2011, p. 3).

Si aplicamos la definición anterior a los Estudios de Traducción, la autoeficacia para traducir, o autoeficacia del traductor, representa la confianza que un traductor o estudiante de traducción tiene en su capacidad para traducir (Haro-Soler, 2017a, 2017b). Dentro de los Estudios de Traducción, la autoeficacia puede clasificarse, por tanto, como un constructo propio de la Psicología de la Traducción, que, tal y como apuntan Jääskeläinen (2012) y Bolaños-Medina (2016), no se refiere únicamente a la investigación del proceso de traducción, sino que se centra en el estudio de factores sociales, conductuales, cognitivos y emocionales del traductor y su interacción con el entorno donde ejerce su actividad, así como con otros agentes que intervienen en dicho entorno, todo ello tanto desde un punto de vista objetivo como desde la percepción de los propios traductores (Bolaños-Medina, 2016, p. 66).

La investigación en torno a la autoeficacia ha sido abundante en un amplio abanico de ámbitos (Bong, 2002, p. 133; Pajares, 1996, p. 545; Torre, 2007, pp. 85-86), entre los que destaca el educativo (Bolaños-Medina, 2014, p. 199; Pajares, 1996, p. 545). El estudio de la autoeficacia en el ámbito académico no debe sorprendernos si tenemos en cuenta los efectos que puede traer consigo para el estudiantado. De acuerdo con la Teoría Social Cognitiva (Bandura, 1987), donde la autoeficacia ocupa una posición central, esta puede influir en la toma de decisiones y en la selección de actividades en las que el estudiante decide participar, incrementar la motivación, favorecer el esfuerzo y la persistencia que se dedican durante el desempeño de la actividad, permitir el control de estados emocionales como el estrés y la ansiedad, que impedirían hacer buen uso de los recursos internos, e identificar vías de resolución de los problemas que surgen en el desempeño de la tarea.

Cabe señalar que, habida cuenta de los efectos mencionados, la autoeficacia se encuentra tras varias de las competencias genéricas que distingue el proyecto Tuning tanto en Europa como en Latinoamérica. Entre estas competencias se encuentran la motivación, la toma de decisiones, y la identificación y resolución de problemas (Beneitone et al., 2007; González \& Wagenaar, 2003), siendo estas últimas especialmente relevantes para la actividad traductora1.

A pesar de sus efectos y de la relación que guarda con las competencias mencionadas, en el ámbito de la formación de traductores la autoeficacia ha permanecido en un segundo plano (Atkinson \& Crezee, 2014), probablemente debido a la dificultad que supone plasmar en objetivos didácticos competencias relacionadas con la psicología del traductor (Presas, 1998, p. 134; Way, 2014, p. 143). El vacío que existe en la formación de traductores en cuanto al objetivo de contribuir de forma explícita y estructurada al desarrollo de la confianza del estudiantado en sus capacidades para traducir no solo ha sido identificado por el colectivo de investigadores/profesorado, sino también por el estudiantado

1 En este sentido, Pym (1992) indica que la traducción “is a process of generation and selection, a problem-solving process that often occurs with apparent automatism". 
(Haro-Soler, 2017a). Con el fin de arrojar luz sobre posibles modos de abordar este objetivo didáctico, resolvimos diseñar e implementar una experiencia formativa destinada a favorecer el desarrollo de la autoeficacia del estudiantado. Dicha experiencia formativa se materializó en un taller de dos horas de duración que se llevó a cabo en la asignatura Traducción 1 C-A (inglés-español), perteneciente al Grado en Traducción e Interpretación de la Universidad de Granada (España).

En el presente artículo expondremos los fundamentos teóricos en los que se apoyó esta experiencia, describiremos su puesta en práctica y presentaremos los resultados de un estudio empírico-descriptivo que corresponde a la evaluación que el estudiantado participante realizó de esta experiencia. Para recoger la opinión de los participantes en cuanto a la utilidad y pertinencia del taller organizado se recurrió a un cuestionario en línea que ha arrojado datos de corte tanto cualitativo como cuantitativo.

Conviene especificar que, si bien la acción formativa descrita en las páginas que siguen se dirigió a estudiantes del Grado en Traducción e Interpretación, puede adaptarse y extrapolarse a otras disciplinas con facilidad, en tanto que se trata de una experiencia concebida para ser implementada en una etapa inicial del proceso de desarrollo de la autoeficacia y funciona, por tanto, como los cimientos a partir de los que el estudiantado construirá su autoeficacia.

\section{DISEÑO DE LA EXPERIENCIA FORMATIVA: FUNDAMENTOS TEÓRICOS ¿Qué es la autoeficacia?}

Como ya se ha indicado en la introducción del presente trabajo, la autoeficacia se define como "la confianza que tiene una persona de que posee la capacidad para hacer las actividades que trata de hacer" (Blanco et al., 2011, p. 3). Consiste, por tanto, en una autopercepción sobre las propias capacidades que se construye en función de la tarea o actividad concreta que se pretende desarrollar len nuestro caso, la traducción). Es precisamente su especificidad con respecto a una actividad particular (Bandura, 1987, pp. 421-422, 1997, pp. 36-42, 2006, p. 307) lo que distingue la autoeficacia de conceptos pertenecientes al mismo campo semántico, como el de autoconfianza, que Schunk y DiBenedetto (2016, p. 40) definen como: "a general capability self-belief that often fails to specify the object of the belief". Nos encontramos en este caso ante una autopercepción referente a las capacidades generales que se poseen y que no está vinculada a ninguna actividad concreta.

La diferencia entre la autoconfianza y la autoeficacia implica que un estudiante que considera que, en general, puede hacer frente a una amplia variedad de situaciones en su vida diaria gracias a las capacidades que posee (estudiante con autoconfianza), puede carecer de confianza en sus capacidades para traducir (estudiante con baja autoeficacia para traducir). En sentido contrario, un estudiante que confíe en sus capacidades para resolver problemas matemáticos (alta autoeficacia para las matemáticas) puede carecer de autoconfianza al considerar que, en general, no posee las capacidades necesarias para enfrentarse a situaciones de diversa naturaleza en su día a día (Haro-Soler, 2017a).

Si bien es cierto que el término autoconfianza está más extendido en el uso diario, probablemente por la similitud del vocablo con su significado; desde el punto de vista empírico la especificidad de la 
autoeficacia le otorga mayor valor predictivo (Bandura, 1987, 1997, 2006; Salanova, Peiró \& Schaufeli, 2002). Asimismo, la vinculación de la autoeficacia con la actividad concreta para la que se requieren las capacidades que son objeto de dicha autopercepción facilita en cierta medida la observación de los comportamientos en los que se manifiesta, así como el diseño e implementación de prácticas docentes para su desarrollo.

Una vez que hemos delimitado el objeto de la experiencia formativa que nos ocupa, estamos en disposición de analizar el armazón teórico del que partimos para diseñar esta experiencia. De este modo, analizaremos las fuentes y efectos de la autoeficacia según la teoría de la que forma parte, la Teoría Social Cognitiva (Bandura, 1987). Aunque no cabe duda de la pertinencia de asentar el diseño de nuestra experiencia formativa sobre las fuentes de la autoeficacia, conviene especificar que se describirán igualmente sus efectos, puesto que, tal y como se explicará a continuación, algunos de ellos poseen la doble naturaleza de causa-efecto. Asimismo, revisaremos prácticas docentes propuestas por diversos autores para desarrollar la autoeficacia del estudiantado.

\section{Teoría Social Cognitiva: fuentes y efectos de la autoeficacia}

La autoeficacia ocupa una posición central en la Teoría Social Cognitiva de Bandura (1987), que la concibe como un tipo de autoconocimiento que resulta de la capacidad de autorreflexión del ser humano (Bandura, 1997, p. 79). Más concretamente, la autoeficacia se origina a partir de cuatro fuentes de información que son procesadas a través del pensamiento autorreferente: la experiencia directa, el aprendizaje vicario, la persuasión verbal y estados fisiológicos y emocionales (Bandura, 1997).

La experiencia directa constituye la principal fuente de información a partir de la que se conforma la autoeficacia. En general, los éxitos o logros de ejecución favorecen la confianza que se posee en las propias capacidades, mientras que los fracasos llevan a dudar de la propia competencia (Bandura, 1997, p. 80; Pajares, 1997, p. 4). El aprendizaje vicario, o la experiencia de personas que se perciben como modelos, también repercute en la autoeficacia. De esta forma, los éxitos de aquellos que se perciben como modelos pueden incrementar la autoeficacia, mientras que los fracasos del modelo pueden provocar una disminución de la confianza que se posee en las propias capacidades para llevar a cabo la tarea que el modelo no ha podido completar (Bandura, 1997, pp. 86-87).

Por su parte, la persuasión verbal ha de entenderse como "lo que los otros nos dicen acerca de nuestro desempeño" (Prieto, 2007, p. 85). El hecho de que los que nos rodean confíen en nuestra capacidad para llevar a cabo una tarea concreta favorece la autoeficacia, mientras que las dudas de terceros sobre nuestra competencia pueden disminuir la confianza que poseemos en nuestras capacidades (Bandura, 1997, p. 101). Cabe subrayar que la persuasión verbal debe versar sobre las capacidades reales del sujeto, pues de no ser así la experiencia directa negativa posterior pondrá fin al incremento impreciso de la autoeficacia que la persuasión verbal había provocado (Bandura, 1997, p. 101; Schunk \& DiBenedetto, 2016, p. 37).

Los estados fisiológicos y emocionales que un individuo experimenta durante el desempeño de una actividad constituyen la cuarta fuente de la autoeficacia. En este sentido, la presencia de emociones 
como el estrés o la aparición de estados fisiológicos como el cansancio o el dolor durante la realización de una tarea, más aún cuando esta requiere esfuerzo físico o control emocional, es interpretada por el individuo como un signo de vulnerabilidad y de incapacidad y, por ende, disminuye la autoeficacia (Bandura, 1997, p. 106).

En cuanto a sus efectos, según la Teoría Social Cognitiva y estudios posteriores, las personas tienden a seleccionar aquellas situaciones y tareas que se consideran capaces de dominar, evitando aquellas otras que, en su opinión, exceden sus capacidades (Bandura, 1997, pp. 160-161). Asimismo, quienes se juzgan eficaces suelen dedicar más esfuerzo y persisten durante más tiempo para hacer frente a las dificultades que surgen en el desempeño de la tarea que aquellos que carecen de confianza en sus capacidades (Bandura, 1987, p. 419).

La autoeficacia repercute igualmente en los patrones de pensamiento y reacciones emocionales del individuo, de forma que quienes dudan de sus capacidades para llevar a cabo una tarea concreta tienden a pensar que esta es más complicada de lo que realmente es, lo cual provoca estrés y ansiedad. Estos dos estados emocionales dificultan la utilización adecuada de los recursos internos e impiden desarrollar una visión clara sobre el mejor modo de resolver los problemas que surgen en el desempeño de la tarea (Bandura, 1997, pp. 140-141). Nótese, además, que, tal y como se ha explicado anteriormente, la aparición de estos estados emocionales se percibe como un signo de vulnerabilidad e incapacidad, lo cual repercute negativamente en la autoeficacia.

Finalmente, la autoeficacia deja sentir su influencia en la motivación y en la representación de las metas que se desean alcanzar. En este sentido, los individuos que confían en su capacidad para desempeñar una actividad específica se proponen alcanzar metas más ambiciosas que aquellos que dudan de su competencia. Las metas ambiciosas constituyen, a su vez, incentivos que fomentan y mantienen la motivación. Esto lleva a la persona a persistir hasta conseguir dichas metas, lo cual favorece el desarrollo de las competencias que se poseen y la adquisición de nuevas competencias (Bandura, 1997, pp. 116-118). Al mismo tiempo, la consecución de las metas constituiría un logro de ejecución, que, como ya se ha explicado en párrafos anteriores, funcionaría como fuente de la autoeficacia, incrementándola.

\section{Técnicas para el desarrollo de la autoeficacia}

A la hora de diseñar las actividades que constituirían la experiencia formativa que nos ocupa no solo se tomaron en consideración las fuentes y los efectos de la autoeficacia que establece la Teoría Social Cognitiva (Bandura, 1987), sino también los métodos o prácticas docentes que diversos autores proponen con este fin.

Entre dichos métodos se encuentra la explicación teórica del concepto de autoeficacia (Atkinson, 2014, pp. 8-15). Más específicamente, este autor propone exponer en qué consiste la autoeficacia y presentar los principales hallazgos científicos referentes a las fuentes y efectos de esta, de manera que los estudiantes puedan tomar consciencia de su funcionamiento y su importancia. Asimismo, sugiere situar la autoeficacia en un modelo competencial, de modo que los estudiantes la identifiquen como una competencia más que deben desarrollar durante su formación (Atkinson, 2014, p. 9). Si 
bien esta presentación teórica adoptaría la forma de una clase magistral de alrededor de media hora de duración, debe adaptarse a las necesidades de los estudiantes e ir acompañada de numerosos ejemplos prácticos, una presentación PowerPoint o similar y material audiovisual que apoye las ideas principales (Atkinson \& Crezee, 2014).

La explicación teórica daría paso a un debate grupal en el que los estudiantes pueden compartir sus experiencias e ideas sobre la autoeficacia (Atkinson, 2014, p. 10). A través de los dos métodos citados, los estudiantes podrán conocer en qué consiste la autoeficacia y comprender su funcionamiento y relevancia, lo cual les permitirá observar su comportamiento de manera más objetiva y consciente. Ello no dará lugar a un cambio inmediato en su autoeficacia, pero constituye un requisito indispensable para que puedan modificar la confianza que poseen en sus capacidades reales (Atkinson, 2014, p. 10).

El feedback positivo y constructivo es otra de las prácticas docentes que sugiere Atkinson (2014, p. 14) para ayudar al estudiantado a confiar en sus capacidades como traductores. Ello implica adoptar un enfoque de enseñanza-aprendizaje centrado no solo en los errores sino también en los aciertos, que pueden ir acompañados de elogios o puntos extra en la calificación de la tarea. No obstante, a este respecto señala que, como ya se ha indicado, el elogio a las soluciones adoptadas por el estudiantado debe siempre corresponder con las capacidades reales que estos poseen para evitar el desarrollo de creencias de autoeficacia inexactas (Atkinson, 2014, p. 14). Atkinson (2014, p. 8) y Atkinson y Crezee (2014) proponen igualmente recurrir a la autorreflexión para analizar de forma objetiva los puntos fuertes que se poseen y las competencias que es necesario mejorar, lo cual permitirá al estudiantado desarrollar creencias de autoeficacia realistas, es decir, ajustar cuando sea necesario su autoeficacia de acuerdo con sus capacidades reales.

En este sentido, Way (2008) propone la Ficha de Talón de Aquiles como instrumento para ayudar a los estudiantes a tomar consciencia de sus puntos fuertes y débiles y reflexionar sobre las estrategias que pueden llevar a cabo para transformar en puntos fuertes las competencias que deben aun desarrollar. De forma similar, esta autora (Way, 2009) diseñó la Ficha de Gestión de Proyectos, que cada estudiante debe completar tras cada proyecto de traducción realizado y en la que debe indicar las tareas que ha desempeñado, el tiempo que ha invertido para ello y las dificultades encontradas. De esta forma, guiados por el profesorado, los estudiantes pueden tomar consciencia de cómo ha mejorado su rendimiento conforme avanza la asignatura correspondiente, lo cual se refleja en la disminución del tiempo empleado en cada tarea y del número de dificultades encontradas.

El profesorado participante en el estudio llevado a cabo por Haro-Soler (2017b) coincide con Atkinson (2014) y Way $(2008,2009)$ al considerar que el feedback constructivo, la toma de consciencia de las capacidades reales que se poseen, así como la toma de consciencia de la evolución que experimenta el propio rendimiento pueden repercutir positivamente en la autoeficacia del estudiantado. A estas prácticas añadieron, entre otras, la persuasión verbal.

Con el fin de conocer la opinión del otro colectivo implicado en el proceso de enseñanzaaprendizaje, Haro-Soler (2015, 2017a) llevó a cabo un estudio cualitativo para identificar los métodos que, desde el punto de vista del propio estudiantado, contribuyen al desarrollo de la confianza que 
poseen en sus capacidades como traductores. Entre estos métodos se encuentra la persuasión verbal, que, de acuerdo con los estudiantes que participaron en dicha investigación, resulta efectiva tanto cuando procede del profesorado como cuando la llevan a cabo los compañeros.

Los participantes en la citada investigación explicaron igualmente que el aprendizaje vicario es otro de los métodos que había favorecido su autoeficacia durante la formación en traducción. De acuerdo con los estudiantes, el aprendizaje vicario se produjo a través de la identificación con compañeros, así como con antiguos alumnos que se habían incorporado con éxito al mercado laboral. Por un lado, la identificación con compañeros incrementó la autoeficacia de los participantes al comprobar en el aula que todos cometían los mismos errores y que, por tanto, no había razón para sentirse inferior o inseguro. Por otro lado, los participantes explicaron que conocer la trayectoria profesional de antiguos alumnos, a los que percibían como modelos, favoreció su autoeficacia en tanto que interpretaron los logros acometidos por los egresados como posibles logros propios futuros (Haro-Soler, 2017a).

\section{DESCRIPCIÓN DE LA EXPERIENCIA FORMATIVA}

Ha llegado el momento de describir la experiencia formativa que se llevó a cabo en la Facultad de Traducción e Interpretación de la Universidad de Granada con el fin de favorecer el desarrollo de la autoeficacia del estudiantado. Esta experiencia formativa se materializó en un taller de dos horas de duración que se implementó en una de las sesiones de clase de la asignatura Traducción 1 C-A (inglés-español), que se imparte en el tercer curso del Grado en Traducción e Interpretación de la citada institución. Se trata de una asignatura en la que los estudiantes que tienen como primera lengua extranjera francés, alemán o árabe deben traducir por primera vez desde el inglés, que constituye su segunda lengua extranjera, al español. Al tratarse de su primer contacto con la traducción desde su segunda lengua extranjera, los estudiantes experimentan en muchas ocasiones miedo e inseguridad, razón por la cual se decidió organizar el taller que nos ocupa en esta asignatura.

La implementación del taller corrió a cargo de la autora, profesora responsable de la segunda mitad de la asignatura mencionada (3 créditos ECTS) y se celebró el 19 de mayo de 2017. Un total de 15 estudiantes participaron en el taller.

La duración del taller corresponde a la duración de una sesión de clase. Se contempló la posibilidad de incrementar su duración a cuatro horas y, por tanto, extender el taller a dos sesiones de clase. Sin embargo, el limitado número de créditos disponible para satisfacer otros objetivos didácticos establecidos nos llevó a desechar esa posibilidad. Asimismo, dos horas eran suficientes para satisfacer el objetivo principal que se perseguía con este taller, que se concibió para servir como una primera toma de contacto del estudiantado con el concepto de autoeficacia, al que se aproximarían desde una perspectiva tanto teórica como práctica y en un ambiente distendido, donde el estudiante se convierte en el protagonista del proceso de enseñanza-aprendizaje y la interacción en el motor del taller.

Debemos recordar que la autoeficacia ha permanecido relegada a un segundo plano en la formación de traductores (Atkinson \& Crezee, 2014) y, por lo tanto, el taller se dirigía a un grupo de estudiantes 
que probablemente nunca antes había recibido información o reflexionado sobre la autoeficacia, como ocurría con los estudiantes de la misma institución que participaron en el estudio desarrollado por Haro-Soler (2017a).

Dado el bagaje de los participantes y la limitación temporal señalada, no se pretendía con este taller provocar un incremento inmediato de la confianza del estudiantado en sus capacidades reales, sino sentar las bases necesarias para que, partiendo del conocimiento del concepto de autoeficacia, así como del autoconocimiento, dicho cambio pueda producirse (Atkinson, 2014, p. 10). Además, se pretendía llevar a cabo una serie de actividades prácticas que constituyeran el germen o inicio de dicho cambio. Se trata, en definitiva, de una experiencia formativa concebida para ser implementada en una etapa inicial del proceso de desarrollo de la autoeficacia del estudiantado.

Es por ello que, a pesar de haber sido diseñada e implementada en el ámbito de la formación de traductores, respetando así la especificidad de la autoeficacia con respecto una actividad concreta, no debe el lector pensar que no puede extrapolarse, adaptarse y aplicarse con facilidad a otras disciplinas, tal y como se pondrá de manifiesto a continuación, al presentar las actividades que conformaron el taller.

\section{Introducción teórica y debate grupal: ¿qué es la autoeficacia?}

El taller comienza situando a los estudiantes como protagonistas de la acción formativa, para lo cual se les pregunta por sus conocimientos previos sobre el concepto de autoeficacia: si han oído este término antes, si conocen su significado o, en caso contrario, si pueden imaginar a qué se refiere.

Una vez que se ha establecido un breve debate grupal donde los estudiantes exponen sus ideas y especulaciones, es decir, una vez que se ha despertado su interés por conocer este concepto, se ofrece una definición, que se acompaña de uno de los modelos de competencia traductora que incluye la confianza como una de las competencias que todo traductor debe poseer. En este caso se recurrió al modelo propuesto por Kelly $(1999,2000,2002,2005,2007)^{2}$. Siguiendo así la recomendación de Atkinson (2014, p. 9), los estudiantes pudieron comprobar que la confianza en las propias capacidades es una competencia más que se espera de todo traductor.

A continuación se presentaron las tres situaciones en las que los estudiantes pueden encontrarse según la relación que exista entre sus capacidades reales y la confianza que posean en ellas: exceso de confianza, defecto de confianza o equilibrio entre las capacidades reales que poseen como traductores y la confianza que tienen en ellas. Recordemos que los estudiantes son los protagonistas del taller, en tanto que el objetivo de este consiste en sentar las bases a partir de las que puedan construir creencias precisas de autoeficacia. Por ello, en este punto conviene hacerles reflexionar para identificar aquella situación, de las tres expuestas, en la que se encuentran según la confianza que poseen en sus capacidades. Se produce aquí, por tanto, la puesta en práctica del pensamiento autorreferente.

\footnotetext{
2 Debido a que el término autoeficacia se ha incorporado recientemente a los Estudios de Traducción, el modelo competencial citado no se refiere específicamente a la autoeficacia, sino a la autoconfianza del traductor, término empleado tradicionalmente en este campo. Véase Haro-Soler (2017a) para profundizar en este aspecto.
} 


\section{Debate grupal: ¿cuáles son los efectos de la autoeficacia? ¿Y cómo puede desarrollarse?}

Conocer las situaciones que pueden darse en función de la autoeficacia que poseen y tomar consciencia de la situación en la que se encuentran permite iniciar un debate grupal acerca de cuál consideran que es la situación idónea, así como acerca de los efectos que puede traer consigo el poseer un exceso, un defecto o un nivel de confianza acorde con las propias capacidades. Cabe subrayar que en este punto no es el profesor/la profesora quien realiza una explicación teórica de los efectos de la autoeficacia, sino que son los propios estudiantes los que al compartir sus experiencias identifican dichos efectos. El rol del profesor/la profesora en este momento se limita a señalar que las experiencias vividas por los estudiantes tienen respaldo científico y a situarlas como efectos de la autoeficacia dentro de la Teoría Social Cognitiva.

Con respecto a las fuentes, el procedimiento es similar: al preguntar a los estudiantes cómo creen que pueden adquirir confianza en sus capacidades, estos expondrán situaciones vividas a lo largo de su formación. Sin duda han tenido experiencias en las que el feedback positivo o los logros de ejecución han repercutido positivamente en su autoeficacia. En consecuencia, identificarán por ellos mismos las fuentes que establece la Teoría Social Cognitiva.

\section{Actividad práctica 1: análisis objetivo de las propias capacidades y aprendizaje vicario}

Tras los primeros 40 minutos del taller los estudiantes ya están familiarizados con el concepto de autoeficacia, su funcionamiento y su relevancia tanto en el ámbito académico como en el profesional. Llega entonces el momento de implementar varias actividades prácticas destinadas a ayudar a los participantes a tomar consciencia de sus capacidades, lo que les permitirá comenzar a ajustar la confianza que poseen en ellas en caso de ser necesario (Atkinson, 2014, pp. 8-15).

Como parte de la primera actividad se pide a los estudiantes que identifiquen y escriban tres puntos fuertes que posean como traductores ${ }^{3}$. Por sorprendente que pueda parecer, más de la mitad de los estudiantes que participaron en este taller tuvieron grandes dificultades para enumerar tres de sus puntos fuertes, a pesar de contar con buenas capacidades para la traducción, lo cual refleja la necesidad de que analicen objetivamente sus capacidades, tomen consciencia de ellas e incrementen su autoeficacia.

Una vez que todos los estudiantes identificaron sus puntos fuertes, se pidió a varios de ellos que los compartieran con el resto la clase. Recurriendo a la persuasión verbal la profesora reafirmaba las capacidades expuestas individualmente e incluso añadía otras a la lista que cada estudiante había preparado.

A continuación se pidió a los estudiantes que identificaran tres de sus puntos débiles, lo cual les resultó mucho más sencillo habida cuenta de la escasa autoeficacia que poseían. Además de identificar tres capacidades que debían mejorar, se les pidió que escribieran estrategias que podrían llevar a cabo para ello, siguiendo así la propuesta de Way (2008) ya descrita anteriormente. De nuevo,

\footnotetext{
3 Nótese que para adaptar esta actividad a otras disciplinas basta con modificar la tarea para la que se requieren dichos puntos fuertes (redacción, composición musical, expresión gráfica, etc.).
} 
se animó a aquellos estudiantes que lo desearan a que compartieran sus puntos débiles y las vías de mejora que habían identificado con el resto de la clase. Esta puesta en común resulta esencial para favorecer la identificación con otros compañeros (aprendizaje vicario, Bandura, 1997), de modo que los estudiantes comprendan que no son los únicos que cuentan con puntos débiles que deben trabajar (Haro-Soler, 2017a).

Para completar esta actividad y aplicar la autorreflexión y el autoconocimiento a una situación real se presentó una oferta de trabajo extraída del portal InfoJobs. Los estudiantes debían decidir si aceptarían o rechazarían esta oferta tras analizar de manera objetiva sus capacidades como traductores y compararlas con las que se requerían para la oferta presentada.

\section{Actividad práctica 2: persuasión verbal procedente de compañeros}

En la segunda actividad práctica del taller los estudiantes debían trabajar por parejas. Se pidió a los participantes que, en lo posible, se agruparan con compañeros con los que habían trabajado previamente, o con los que habían trabado amistad, dado que cada miembro de la pareja debía identificar tres puntos fuertes con los que su compañero/a contase como traductor/a y hacérselos saber. Esta actividad completaría la persuasión verbal que la profesora ya había comenzado a ejercer en la actividad anterior, si bien en este caso procedería de los compañeros, siguiendo así los hallazgos del estudio desarrollado por Haro-Soler (2017a).

Esta actividad se acompañó de un fragmento de uno de los capítulos de la serie cómica americana Cómo conocí a vuestra madre, donde uno de los personajes protagonistas se convence a sí mismo, recurriendo a la persuasión verbal ante un espejo, de que posee las capacidades necesarias para correr una maratón. De este modo, incorporamos al taller la recomendación de Atkinson y Crezee (2014), que sugieren utilizar material audiovisual en experiencias formativas centradas en la autoeficacia del estudiantado.

\section{Actividad 3: toma de consciencia de la evolución del rendimiento}

Se pidió a los estudiantes que reflexionaran acerca de la evolución que habían experimentado sus capacidades como traductores desde el comienzo de sus estudios en la Facultad de Traducción e Interpretación hasta el momento en el que se encontraban, siguiendo la línea de las propuestas de Way (2009) y Haro-Soler (2017b) ya descritas anteriormente. Esta reflexión vino acompañada de una puesta en común a nivel grupal, continuando con el carácter interactivo del taller, y tuvo como resultado la toma de consciencia por parte de los participantes del desarrollo que había experimentado su competencia traductora a lo largo de los tres últimos años.

\section{Actividad práctica 4: aprendizaje vicario a través de la experiencia de egresados}

La cuarta actividad del taller persigue la identificación de los estudiantes con antiguos alumnos de la titulación que se encuentran cursando y que se han incorporado de forma satisfactoria al mercado laboral. Resulta esencial que los profesionales cuya experiencia se presente estudiaran en el mismo centro y cursaran la misma titulación que los participantes, de modo que los estudiantes puedan percibir a los egresados como modelos e interpreten los logros de estos como posibles logros futuros 
propios (aprendizaje vicario, Bandura, 1997, pp. 86-87). Para presentar la experiencia profesional de los egresados se incluyeron en la presentación PowerPoint que se utilizó en el taller varias diapositivas con el nombre, la foto y los aspectos más relevantes de la formación y experiencia profesional de antiguos alumnos, previa autorización de los implicados.

\section{Actividad 5: establecimiento de objetivos como incentivos motivacionales}

Para comprender el objetivo de esta actividad debemos recordar que, de acuerdo con la Teoría Social Cognitiva de Bandura (1997, pp. 116-118), los individuos que confían en sus capacidades establecen metas más ambiciosas, que funcionan como incentivos motivacionales que les ayudan a no cesar en su empeño hasta alcanzar dicha meta. La consecución del objetivo constituye a su vez un logro de ejecución, que favorece la confianza en las propias capacidades.

Mediante la quinta actividad del taller se pretendía intervenir en dicho ciclo, pidiendo a los estudiantes, especialmente a aquellos que desconfían de sus capacidades, que reflexionaran acerca de a qué les gustaría dedicarse una vez que finalicen su formación, dejando a un lado las dudas y los miedos que pudieran asaltarles. Se les pidió, además, que dibujaran la meta identificada y que conservaran dicho dibujo ${ }^{4}$, de modo que pudiera servirles como fuente de motivación hasta la consecución de la meta establecida, lo cual repercutirá finalmente de forma positiva en su autoeficacia.

\section{Cierre}

Tras dos horas de interacción, aproximación al concepto de autoeficacia, autorreflexión, toma de consciencia, aprendizaje vicario, persuasión verbal y establecimiento de objetivos, se puso fin al taller recordando a los estudiantes que pueden conseguir todo lo que se propongan siempre y cuando conozcan sus capacidades reales, confíen en ellas y continúen trabajando para desarrollar aquellas que deban mejorar. Para cerrar el taller puede proyectarse también alguno de los numerosos vídeos disponibles en línea que versan sobre motivación y confianza.

\section{MÉTODO \\ Diseño}

Finalizada la implementación de la experiencia formativa, resultaba imprescindible conocer la opinión del estudiantado sobre la utilidad y relevancia del taller, más aun teniendo en cuenta que este giraba en torno a los participantes y que su objetivo no era otro que sentar las bases para el desarrollo de su autoeficacia. Consecuentemente, con el fin de recoger las percepciones del estudiantado, se llevó a cabo un estudio empírico-descriptivo basado en la técnica de la encuesta. Esta técnica se materializó en un cuestionario en línea diseñado ad hoc para que los estudiantes evaluaran la experiencia formativa que nos ocupa tanto desde un punto de vista cualitativo como cuantitativo (véase Técnicas de recolección de datos).

\footnotetext{
4 Esta actividad se basó en uno de los talleres que se llevaron a cabo en las Jornadas de Profesionalización celebradas en la Facultad de Traducción e Interpretación de la Universidad de Granada en la primavera de 2012.
} 


\section{Participantes}

Recordemos que un total de 15 estudiantes participaron en el taller. Todos ellos cursaban el tercer curso del Grado en Traducción e Interpretación de la Universidad de Granada y estaban matriculados en la asignatura Traducción $1 \mathrm{C}$-A (inglés-español). Se recurrió por tanto al muestreo no probabilístico por conveniencia (Nunan, 2005, p. 41). Del total de participantes (15), 13 eran mujeres (86,7\%) y solo 2 hombres (13,3\%). De todos ellos, 14 completaron el cuestionario en línea que se les había facilitado. Por tanto se obtuvo una tasa de respuesta elevada, correspondiente al 93,3\%.

\section{Técnicas de recolección de datos}

El cuestionario que se distribuyó a los estudiantes al finalizar el taller se diseñó específicamente para evaluar la experiencia formativa descrita. Con dicho cuestionario se perseguían los siguientes objetivos:

- Conocer la opinión de los participantes en cuanto a la utilidad del taller para su formación como traductores.

- Conocer la importancia que los participantes conceden a este tipo de experiencias y su opinión en cuanto a la incorporación de este tipo de iniciativas a la formación en traducción.

- Conocer aquellos aspectos del taller que los participantes destacan como positivos.

- Conocer aquellos aspectos del taller que los participantes consideran negativos y recoger sugerencias de mejora para su incorporación en futuras ediciones.

- Conocer la opinión de los participantes sobre cualquier otro aspecto relacionado con el taller que desearan comentar.

Para satisfacer dichos objetivos se incluyeron en el cuestionario un total de ocho preguntas (ver Apéndice Al. Se decidió incorporar el mínimo número de preguntas posible en aras de lograr una tasa de respuesta relativamente elevada. Cuatro de ellas eran preguntas cerradas con valores de respuesta de 1 a 4 que reflejan el grado de acuerdo o desacuerdo con los ítems correspondientes. Las cuatro restantes constituían preguntas abiertas con las que se pretendía obtener información cualitativa que permitiera llevar a cabo una interpretación más profunda de los datos cuantitativos obtenidos mediante las preguntas cerradas. El cuestionario se sometió a la valoración de tres jueces. Todos ellos son expertos en el área de diseño de cuestionarios y dos de ellos son también especialistas en Didáctica de la Traducción. El cuestionario se elaboró a través de la aplicación Google Forms, de uso sencillo e intuitivo. Para completarlo bastaba con que los estudiantes accedieran al enlace que se les facilitó, respondiesen a las preguntas en línea y registraran sus respuestas.

\section{Procedimiento}

Como se ha indicado previamente, para diseñar el taller implementado se tomaron en consideración las fuentes y efectos de la autoeficacia que establece la Teoría Social Cognitiva (Bandura, 1987), así como algunas de las prácticas docentes que diversos autores (Atkinson, 2014; Atkinson \& Crezee 2014; Haro-Soler, 2015, 2017a, 2017b; Way, 2008, 2009) proponen con el fin de desarrollar la autoeficacia del estudiantado de traducción. Dicho taller comenzó con una introducción teórica del concepto de autoeficacia, que dio lugar a un debate grupal que permitió a los estudiantes familiarizarse con el 
funcionamiento de esta competencia. Siguieron prácticas tan variadas como la toma de consciencia de los puntos fuertes y débiles que se poseen a la hora de traducir, la persuasión verbal, el aprendizaje vicario o el establecimiento de objetivos que funcionan como incentivos motivacionales. Tras finalizar el taller, se envió un mensaje colectivo a los participantes a través de la plataforma de la asignatura (Prado2) en el que se incluía el enlace al cuestionario. En dicho mensaje se informó de que la compleción del cuestionario era totalmente voluntaria y anónima, además de especificar que los datos obtenidos se utilizarían con fines de investigación.

Una semana más tarde se procedió al análisis de los datos recogidos. Los resultados referentes al grado de acuerdo o desacuerdo con los ítems que constituyen las preguntas cerradas del cuestionario se presentan en forma de porcentajes para cada una de las opciones de respuesta. En cuanto a la información cualitativa recogida por medio de las preguntas abiertas se procedió a su categorización y codificación (Suárez, 2005, p. 102), es decir, a su clasificación en las categorías que se identificaron para cada uno de los objetivos del estudio tras la lectura analítica de las respuestas obtenidas. Dichas categorías son: autorreflexión, aproximación al concepto de autoeficacia (fuentes y efectos), incorporación de talleres centrados en la autoeficacia a la formación en traducción, interacción, enfoque de enseñanza-aprendizaje centrado en el estudiante, aprendizaje vicario a través de compañeros, aprendizaje vicario a través de egresados, persuasión verbal, establecimiento de objetivos, duración del taller, contenido del taller.

\section{RESULTADOS}

\section{Utilidad del taller para la formación en traducción}

Ante el ítem "El taller me ha parecido útil para mi formación como traductor/a" el 64,3\% de los participantes indicaron estar totalmente de acuerdo y el 35,7\% restante optaron por la opción "de acuerdo". De este modo, la totalidad de los participantes del taller considera que este repercute positivamente en su formación como traductores.

Al preguntar a los participantes por las razones por las que consideran que el taller resulta útil para su formación predominan las respuestas que relacionan la utilidad del taller con la importancia de la autorreflexión y de la toma de consciencia de las capacidades que se poseen:

Me ha ayudado a reflexionar sobre cómo me siento con mi carrera y mi futuro.

Porque es bueno conocerse para poder ser buenos profesionales.

Es bueno reflexionar sobre la traducción, las posibilidades y también sobre las capacidades de cada uno. Me ha parecido interesante.

Nos ayuda a valorarnos más, a tener más confianza y nos anima a luchar por nuestros objetivos. 
Asimismo, son frecuentes las respuestas que hacen referencia a la importancia de conocer las fuentes a partir de las que se genera la autoeficacia y los efectos que tener un exceso, un defecto o un nivel de confianza acorde con las capacidades que se poseen puede traer consigo:

Porque he aprendido que tener una buena confianza en uno mismo, sin sobrepasarse, puede ayudar a traducir y tomar decisiones más contundentes.

Porque muchas veces no somos conscientes de cómo nuestra inseguridad nos puede afectar a la hora de traducir.

Es bueno detenerse y analizar mi situación personal en cuanto a mi motivación y comprobar qué posibles medidas puedo tomar de cara a los problemas que pueda encontrar en relación a mi confianza.

El hecho de que los estudiantes justifiquen la utilidad del taller en base a la importancia de conocer el concepto de autoeficacia, sus fuentes y efectos, y de tomar consciencia de las capacidades que se poseen mediante el pensamiento autorreferente parece indicar que, tal y como apunta Atkinson (2014, pp. 8-15), aproximarse de forma teórica al concepto de autoeficacia y comprender su funcionamiento es un paso esencial para comenzar a observar el propio comportamiento de forma más consciente, analizar las propias capacidades de forma objetiva y, de ser necesario, llevar a cabo cambios en el nivel de confianza que se posee en ellas.

En consecuencia, las respuestas cualitativas obtenidas parecen reflejar que se ha satisfecho el objetivo principal que se perseguía con el taller, que consistía en sentar las bases necesarias para que, mediante la familiarización con el concepto de autoeficacia y mediante el autoconocimiento, los participantes puedan modificar la confianza que poseen en sus capacidades como traductores de resultar necesario.

Para asegurarnos de que los participantes completaban el cuestionario con atención y para triangular su opinión sobre la utilidad del taller, se incluyó el siguiente ítem negativo en el cuestionario: "Talleres como este son una pérdida de tiempo", con el que 12 participantes expresaron estar totalmente en desacuerdo $(85,7 \%)$, un estudiante indicó estar en desacuerdo $(7,1 \%)$ y otro estudiante indicó estar totalmente de acuerdo $(7,1 \%)$. Sin embargo, las respuestas cualitativas de este último estudiante, en las que subraya la utilidad del taller y la importancia de incorporarlo a la formación en traducción, nos llevan a pensar que no invirtió la escala de grado de acuerdo o desacuerdo tal y como debería haber hecho al tratarse de un ítem negativo y seleccionó, por tanto, la opción "totalmente de acuerdo" en lugar de "totalmente en desacuerdo". No obstante, al tratarse de un cuestionario anónimo no se pudo contactar con el participante en cuestión para confirmar o rechazar esta suposición.

\section{Incorporación de talleres similares a la formación en traducción}

El $100 \%$ de los participantes en el taller indicaron estar totalmente de acuerdo $(78,6 \%)$ o de acuerdo $(21,4 \%)$ con el ítem “Me gustaría que este tipo de talleres formaran parte del Grado en Traducción e 
Interpretación". Además, la totalidad de los participantes señaló estar totalmente de acuerdo $(78,6 \%) 0$ de acuerdo $(21,4 \%)$ con el ítem "Me gustaría que este tipo de talleres se organicen en más asignaturas".

En este sentido conviene señalar que tanto en la pregunta en la que los estudiantes podían indicar sugerencias de mejora, como en la última pregunta del cuestionario, en la que podían incluir cualquier comentario relacionado con el taller que desearan expresar; se recogieron numerosos comentarios instando a la incorporación de este tipo de iniciativas al Grado en Traducción e Interpretación, bien mediante una asignatura independiente destinada a tratar aspectos relacionados con la psicología del traductor, bien mediante la celebración de talleres similares en otras asignaturas. El último comentario va más allá de la titulación citada y sugiere incluso la incorporación de este tipo de experiencias formativas a otras disciplinas:

Implantar más talleres, ejercicios prácticos e incluso una asignatura que trate todos estos aspectos y nos ayude a formarnos como traductores en el ámbito personal.

Que se repita durante la carrera más veces un taller así, porque a veces los estudiantes se vienen abajo y necesitan que alguien los anime.

Me parece una iniciativa muy bonita y totalmente necesaria que debería tener más importancia en el plan de estudios de nuestro grado.

Me gustaría que nos hicieran más talleres de este tipo.

Hacerlo en más asignaturas y que se siga haciendo.

[...] una asignatura de este tipo no solo sería muy beneficiosa para el Grado de Traducción, sino para cualquier otra carrera y nivel de estudios.

\section{Aspectos positivos del taller}

Se incluyó en el cuestionario una pregunta abierta en la que se pedía a los estudiantes que expresaran su opinión acerca de los aspectos del taller que consideran positivos o dignos de mención. En línea con los comentarios que los participantes incluyeron en preguntas anteriores para justificar la utilidad que el taller organizado tiene para su formación, se registraron en esta pregunta varios comentarios que señalaban la reflexión sobre las propias capacidades como uno de los aspectos más relevantes del taller:

Reflexionar sobre nuestros puntos fuertes y decirle al compañero sus puntos fuertes.

La reflexión, las capacidades, las habilidades.

El modo de reflexionar. 
Me parece que es importante que reflexionemos sobre nosotros mismos y nos valoremos, especialmente en estas épocas en las que acecha el extremo cansancio.

[...] hacerme pensar en dos puntos fuertes como traductora.

El hecho de plantearnos cómo nos sentíamos al principio y como nos sentimos ahora.

De igual modo, se registraron varios comentarios que destacaban la aproximación teórica al concepto de autoeficacia que se realizó al comienzo del taller:

La importancia de la seguridad en uno mismo para poder realizar correctamente nuestra labor.

Ver que para ser un buen traductor tanto la confianza como tus capacidades deben de estar igualadas porque si no es así no se podrá hacer una buena traducción.

Subrayar la importancia de la confianza, pero advertir de los peligros del exceso de esta.

Lo que más positivo me ha parecido ha sido hacer la comparación de cómo afecta al traductor tener mucha confianza y pocas cualidades, poca confianza y muchas cualidades y cómo sería poseer ambas en la misma medida.

De nuevo, el hecho de que los estudiantes destaquen como aspectos positivos la reflexión sobre las propias capacidades y el funcionamiento del concepto de autoeficacia parece indicar que se ha satisfecho el objetivo principal que perseguíamos con este taller: establecer los cimientos necesarios para que, a partir del autoconocimiento y de la comprensión del funcionamiento del concepto de autoeficacia, sus fuentes y efectos (Atkinson, 2014, pp. 8-15), los participantes pudieran construir sus creencias de autoeficacia.

Por añadidura, se han registrado comentarios que destacan el carácter interactivo del taller y que confirman que, tal y como se estableció desde su diseño, la interacción constituyó el motor de esta experiencia formativa:

Interactuar con los compañeros y compartir ideas y opiniones. Que no sea una charla; se nos da la oportunidad de expresarnos. Contar testimonios reales de otras personas.

Que sea colectivo. Hablar de los temas del taller con los compañeros ayuda a comprender una situación generaly a darte cuenta de que no eres laúnica persona que puede sentirse poco motivada.

Uno de los participantes destaca igualmente el enfoque de enseñanza-aprendizaje que se decidió adoptar en el taller, donde el estudiante se convertía en el centro de la experiencia formativa: 
En la mayoría de las asignaturas únicamente se centran en aprobar el examen y quizás no se centran tanto en este tipo de aspectos que son fundamentales para nuestra formación, pues también de nuestro ánimo, confianza y seguridad parte nuestro posterior esfuerzo y dedicación a la hora de trabajar/estudiar.

Asimismo, varios participantes hicieron referencia al aprendizaje vicario (Bandura, 1997) como aspecto positivo del taller. Concretamente, destacan la oportunidad de conocer la experiencia de antiguos alumnos de la titulación (identificación con egresados) y de comprobar que todos los compañeros se encuentran en la misma situación (identificación con compañeros), lo cual coincide con los resultados obtenidos en estudios anteriores (Haro-Soler, 2017a).

[...] me ha gustado saber que otros alumnos de la UGR han conseguido objetivos.

Que sea colectivo. Hablar de los temas del taller con los compañeros ayuda a comprender una situación general y a darte cuenta de que no eres la única persona que puede sentirse poco motivada.

Contar testimonios reales de otras personas [profesionales].

Finalmente, encontramos también comentarios donde los participantes presentan como aspectos destacables del taller la persuasión verbal (Bandura, 1997, p. 101) y el establecimiento de objetivos, que funcionan como incentivos motivacionales (Bandura, 1997, pp. 116-118) y favorecen la autoeficacia tras la consecución de la meta establecida:

Reflexionar sobre nuestros puntos fuertes y decirle al compañero sus puntos fuertes [persuasión verbal].

Que nos ayude a visualizar lo que queremos ser en el futuro cuando acabemos nuestra carrera, y tener clara nuestra meta.

Nos ayuda a valorarnos más, a tener más confianza y nos anima a luchar por nuestros objetivos.

Si comparamos los comentarios expuestos en este apartado con las prácticas docentes y las actividades que constituyen la estructura del taller, que recordemos están basadas en la Teoría Social Cognitiva (Bandura, 1987, 1997) y en estudios posteriores (Atkinson, 2014; Atkinson \& Crezee, 2014; Haro-Soler, 2015, 2017a, 2017b; Way, 2008, 2009); podemos comprobar que los participantes han identificado como aspectos destacables del taller todas y cada una de estas prácticas y actividades. Asimismo, consideran positivo el enfoque de enseñanza-aprendizaje adoptado y el carácter interactivo con el que se concibió el taller: 
Tabla 1

Aspectos positivos del taller en opinión de los participantes

Esqueleto del taller
Aspecto positivo del taller según los participantes

Familiarización con el concepto de autoeficacia, sus fuentes y efectos (mediante la exposición teórica y el debate grupal) Toma de consciencia de las capacidades lautorreflexión y autoconocimientol

Aprendizaje vicario a través de compañeros

Aprendizaje vicario a través de egresados

Persuasión verbal

Establecimiento de objetivos como incentivos motivacionales

El estudiante como protagonista del taller

Interacción, debates grupales

sí

\section{Aspectos negativos del taller y sugerencias de mejora}

En cuanto a los aspectos del taller que conviene revisar para su implementación en futuras ocasiones, los comentarios registrados hacen referencia a su duración limitada, además de expresar el deseo de los participantes de ampliar el contenido del taller e incluir, junto a la autoeficacia, otras competencias relacionadas con la psicología y el aprendizaje:

Que sea más largo, y más profundo. Con otros temas sobre el aprendizaje y cómo mejorar nuestro nivel durante la carrera en caso de no tener el nivel necesario.

Aunque el tiempo del que disponíamos no era el suficiente, dentro de la capacidad psicofisiológica encuentro especialmente importante, además de la confianza, la capacidad de concentración. Algún taller enfocado a este tema sería interesante, ya que, en lo personal, suelo tener problemas de esta índole.

A estas sugerencias hay que añadir los comentarios en los que los estudiantes instaban a la incorporación de este tipo de talleres en su formación, bien en forma de asignatura independiente, o mediante su organización en otras asignaturas. Las sugerencias presentadas, por tanto, no hacen sino respaldar la necesidad de cubrir el vacío existente en la formación de traductores en cuanto al desarrollo de la autoeficacia en particular y de otras competencias psicológicas en general (Atkinson \& Crezee, 2014; Presas, 1998, p. 134; Way, 2014, p. 143), así como la necesidad de llevar a cabo experiencias formativas que, como las descritas en estas páginas, contribuyan a cubrir dicho vacío. 


\section{DISCUSIÓN}

En vista del vacío que existe en el ámbito de la formación de traductores en cuanto a la autoeficacia (o confianza en las propias capacidades para traducir) del estudiantado y habida cuenta de los efectos que la autoeficacia puede traer consigo de acuerdo con la Teoría Social Cognitiva (Bandura, 1987, 1997), decidimos diseñar e implementar la experiencia formativa presentada en este artículo. Dicha experiencia perseguía el objetivo principal de sentar las bases necesarias sobre las cuales el estudiantado pudiera construir su autoeficacia, siguiendo para ello un enfoque de enseñanza-aprendizaje centrado en el estudiante y presidido por la interacción.

Tomando como punto de partida los fundamentos teóricos que establece la Teoría Social Cognitiva, así como las prácticas docentes que proponen diversos autores para favorecer la autoeficacia del estudiantado de traducción (Atkinson, 2014; Atkinson \& Crezee, 2014; Haro-Soler, 2015, 2017a, 2017b; Way, 2008, 2009), diseñamos la experiencia formativa referida. Esta experiencia se materializó en un taller de dos horas de duración que se impartió en una de las asignaturas del Grado en Traducción e Interpretación de la Universidad de Granada y que incluyó prácticas tan variadas como la autorreflexión, la toma de consciencia de las propias capacidades, el aprendizaje vicario, la persuasión verbal, el establecimiento de objetivos como incentivos motivacionales o la aproximación al concepto de autoeficacia mediante la explicación teórica y el debate grupal.

Además de describir las actividades que constituyeron dicha experiencia, se han presentado los resultados cuantitativos y cualitativos obtenidos mediante el cuestionario que los participantes en el taller completaron para evaluar la experiencia formativa que nos ocupa. Los resultados reflejan que los participantes consideran que el taller resulta de utilidad para su formación como traductores y estiman que este tipo de experiencias formativas, destinadas a favorecer su autoeficacia, deberían incorporarse a la formación que se encuentran cursando, bien en forma de una asignatura independiente, bien mediante la organización de talleres similares en un mayor número de asignaturas. Asimismo, los estudiantes valoraron como positivas las prácticas docentes y actividades que conformaron el esqueleto del taller, así como el enfoque de enseñanza-aprendizaje adoptado, centrado en el estudiante y presidido por la interacción. Entre las sugerencias de mejora se encuentran el aumento de la duración del taller y la ampliación de su contenido a otras competencias relacionadas con el aprendizaje y la psicología del traductor.

Todo ello pone de manifiesto la necesidad de cubrir el vacío identificado en torno a la autoeficacia y a otras competencias psicológicas del traductor y respalda la implementación de iniciativas como la experiencia formativa que nos ocupa. 


\section{REFERENCIAS}

Atkinson, D. P. (2014). Developing Psychological Skill for the Global Language Industry: An Exploration of Approaches to Translator and Interpreter Training. Translation Spaces, 3(1), 1-24. doi: http://doi.org/10.1075/ts.3.01atk

Atkinson, D. P. \& Crezee, I. H. M. (2014). Improving Psychological Skill in Trainee Interpreters. International Journal of Interpreter Education, 6(1), 3-18. Recuperado de https://goo.gl/w2Cyn8

Bandura, A. (1977). Self-Efficacy: Towards a Unifying Theory of Behavioral Change. Psychological Review, 84(2), 191-215. doi: http://doi. org/10.1016/0146-6402(78)90002-4

Bandura, A. (1987). Pensamiento y acción. Barcelona: Martínez Roca.

Bandura, A. (1997). Self-Efficacy: The Exercise of Control. Nueva York: Freeman.

Bandura, A. (2006). Guide for Constructing Self-Efficacy Scales. En F. Pajares \& T. Urdan (Eds.), Self-Efficacy Beliefs of Adolescents (pp. 307337). Greenwich, CT: Information Age Publishing.

Beneitone, P., Esquetini, C., González, J., Marty, M., Siufi, G. \& Wagenaar, R. (2007). Reflexiones y perspectivas de la Educación Superior en América Latina. Informe Final-Proyecto Tuning-América Latina 2004-2007. Bilbao, Groningen: Universidad de Deusto, Universidad de Groningen. Recuperado de https://goo.gl/41opNj

Blanco, H., Martínez, M., Zueck, M. C. \& Gastélum, G. (2011). Análisis psicométrico de la escala autoeficacia en conductas académicas en universitarios de primer ingreso. Revista electrónica actualidades investigativas en educación, 11(3), 1-27. doi: http://doi.org/10.15517/aie. v11i3.10214

Bolaños-Medina, A. (2014). Self-Efficacy in Translation. Translation and Interpreting Studies, 9(2), 197-218. doi: http://doi.org/10.1075/ tis.9.2.03bol

Bolaños-Medina, A. (2016). Translation Psychology within the Framework of Translator Studies: New Research Perspectives. En C. Martín de León \& V. González-Ruíz (Eds.), From the Lab to the Classroom and Back Again. Perspectives on Translation and Interpreting Training (pp. 59-100). Fráncfort: Peter Lang.

Bong, M. (2002). Predictive Utility of Subject-, Task-, and Problem-Specific Self-Efficacy Judgments for Immediate and Delayed Academic Performances. Journal of Experimental Education, 70(2), 133-162. doi: http://doi.org/10.1080/00220970209599503

González, J. \& Waagenar, R. (Eds.). (2003). Tuning Educational Structures in Europe. Final Report. Phase One. Recuperado de https://goo.gl/ LDbSmj

Haro-Soler, M. M. (2015). La competencia psicofisiológica en el Grado en Traducción e Interpretación: Agentes que influyen en su adquisición. En G. Corpas Pastor, M. Seghiri Domínguez, R. Gutiérrez Florido \& M. Urbano Mendaña (Eds.), Actas del VII Congreso Internacional de la AIETI. Nuevos horizontes en los Estudios de Traducción e Interpretación (pp. 339-351). Recuperado de https://goo.gl/UPsMYd

Haro-Soler, M. M. (2017a). Self-Confidence and its Role in Translator Training: The Students' Perspective. En I. Lacruz \& R. Jääskeläinen (Eds.), New Directions in Cognitive and Empirical Translation Process Research. ATA Series, John Benjamins.

Haro-Soler, M. M. (2017b). Teaching Practices and Translation Students' Self-Efficacy: A Qualitative Study of Teachers' Perceptions. Current Trends in Translation Teaching and Learning, 4(1).

Jääskeläinen, R. (2012). Translation Psychology. En Y. Gambier \& L. van Doorslaer (Eds.), Handbook of Translation Studies III (pp. 191-197). Ámsterdam/Filadelfia: John Benjamins.

Kelly, D. (1999). Proyecto docente e investigador. Granada: Universidad de Granada.

Kelly, D. (2000). Text Selection for Developing Translator Competence. Why Texts From The Tourist Sector Constitute Suitable Material. En C. Schaeffner \& B. Adab (Eds.), Developing Translation Competence (pp. 157-167). Ámsterdam, Filadelfia: John Benjamins.

Kelly, D. (2002). Un modelo de competencia traductora: Bases para el diseño curricular. Puentes, 1, 9-21. Recuperado de https://goo.gl/JGZowu Kelly, D. (2005). A Handbook for Translator Trainers. Translation Practices Explained. Manchester: St. Jerome.

Kelly, D. (2007). Translator Competence Contextualized. Translator Training in the Framework of Higher Education Reform: In Search of Alignment in Curricular Design. En D. Kenny \& K. Ryou (Eds.), Across Boundaries: International Perspectives on Translation Studies (pp. 128142). Cambridge: Cambridge Scholars Publishing.

Nunan, D. (2005). Reserarch Methods in Language Learning. Cambridge: Cambridge University Press.

Pajares, F. (1996). Self-Efficacy Beliefs in Academic Settings. Review of Educational Research, 66(4), 543-578. doi: http://doi. org/10.3102/00346543066004543

Pajares, F. (1997). Current Directions in Self-Efficacy Research. En M. L. Maehr \& P. R. Pintrich (Eds.), Advances in Motivation and Achievement. Vol. 10 (pp.1-49). Greenwich: JAI Press. 
Presas, M. (1998). Los componentes de la competencia pretraductora en el marco del diseño curricular. En I. García Izquierdo \& J. Verdegal (Eds.), Estudios de Traducción: Un reto didáctico (pp. 131-134). Castelló de la Plana: Universitat Jaume I.

Prieto, L. (2007). Autoeficacia del profesor universitario: Eficacia percibida y práctica docente. Madrid: Narcea.

Pym, A. (1992). Translation Error Analysis and the Interface with Language Teaching. En C. Dollerup \& A. Loddegaard (Eds.), Teaching Translation and Interpreting. Training, Talent and Experience (pp. 279-290). Ámsterdam: John Benjamins.

Salanova, M., Peiró, J. M. \& Shaufeli, W. E. (2002). Self-Efficacy Specificity and Burnout among Information Technology Workers: An Extension of the Job Demand-Control Model. European Journal of Work and Organizational Psychology, 11(2), 1-25. http://doi. org/10.1080/13594320143000735

Schunk, D. H. \& DiBenedetto, M. K. (2016). Self-Efficacy Theory in Education. En K. R. Wentzel \& A. Wigfield (Eds.), Handbook of Motivation at School (pp. 35-54). Londres, Nueva York: Routledge.

Suárez, M. (2005). El grupo de discusión: Una herramienta para la investigación cualitativa. Barcelona: Laertes.

Torre, J. C. (2007). Una triple alianza para un aprendizaje universitario de calidad. Madrid: Universidad Pontificia Comillas.

Way, C. (2008). Systematic Assessment of Translator Competence: In Search of Achilles' Heel. En J. Kearns (Ed.), Translator and Interpreter Training: Issues, Methods and Debates (pp. 88-103). Londres: Continuum.

Way, C. (2009). Bringing Professional Practices into Translation Classrooms. En I. Kemble (Ed.), The Changing Face of Translation (pp. 131-142). Portsmouth: University of Portsmouth.

Way, C. (2014). Structuring a Legal Translation Course: A Framework for Decision-Making in Legal Translator Training. En L. Cheng, K. K. Sin \& A. Wagner (Eds.), The Ashgate Handbook of Legal Translation (pp. 135-152). doi: http://doi.org/10.4324/9781315612706

(c) Los autores. Este artículo es publicado por la Revista Digital de Investigación en Docencia Universitaria del Área de Institutional Research and Effectiveness de la Dirección de Aseguramiento de la Calidad, Universidad Peruana de Ciencias Aplicadas. Este es un artículo de acceso abierto, distribuido bajo los términos de la LicenciaCreativeCommons Atribución-Compartirlgual 4.0 Internacional.I http://creativecommons.org/licenses/bysa/4.0/l, que permite el uso no comercial, distribución y reproducción en cualquier medio, siempre que la obra original sea debidamente citada. 


\section{APÉNDICE A}

\section{TALLER SOBRE LA CONFIANZA DEL TRADUCTOR}

Por favor, llene este cuestionario marcando la opción que mejor refleje su opinión. Es importante que no dejes ninguna pregunta en blanco. No te llevará más de cinco minutos. Gracias por tiu colaboració!

1. Este taller me ha parecido útil para mi formación como traductor/a Marca solo un óvalo.

1 (totalmente de acuerdo)

2 (en desacuerdo)

3 (de acuerdo)

4 (Totalmente de acuerdo)

\section{2. ¿Por qué?}

3. Me gustaría que este tipo de talleres formaran parte del Grado en Traducción e Interprestación Marca solo un óvalo.
1 (totalmente de acuerdo)
2 (en desacuerdo)
3 (de acuerdo)
$\longrightarrow 4$ (Totalmente de acuerdo) 
4. Me gustaría que este tipo de talleres se organizaran en más asignaturas Marca solo un óvalo.

1 (totalmente de acuerdo)
2 (en desacuerdo)
3 (de acuerdo)
4 (Totalmente de acuerdo)

5. Talleres como este son una pérdida de tiempo Marca solo un óvalo.

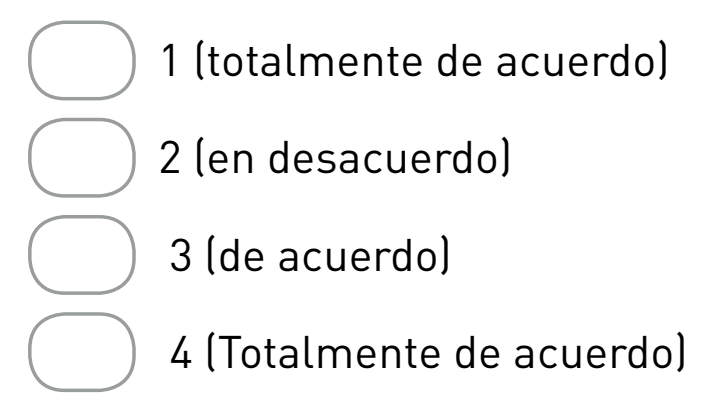

6. Señala aquellos aspectos del taller que hayan parecido positivos

7. Señala aquellos aspectos del taller que consideres negativos e indica sugerencias de mejora 


\section{Otros comentarios}

Has llegado al final del cuestionario! 